\title{
Strong spin-orbit coupling in the noncentrosymmetric Kondo lattice
}

\author{
A. Generalov, ${ }^{1}$ J. Falke, ${ }^{2}$ I. A. Nechaev, ${ }^{3}$ M. M. Otrokov, ${ }^{3,4}$ M. Güttler, ${ }^{2}$ A. Chikina, ${ }^{5}$ K. Kliemt, ${ }^{6}$ S. Seiro, ${ }^{7}$ K. Kummer, ${ }^{8}$ \\ S. Danzenbächer, ${ }^{2}$ D. Usachov, ${ }^{9}$ T. K. Kim, ${ }^{10}$ P. Dudin, ${ }^{10}$ E. V. Chulkov, $, 3,4,11,12$ C. Laubschat, ${ }^{2}$ \\ C. Geibel, ${ }^{13}$ C. Krellner, ${ }^{6}$ and D. V. Vyalikh ${ }^{11,12,14, *}$ \\ ${ }^{1}$ MAX IV Laboratory, Lund University, Box 118, 22100 Lund, Sweden \\ ${ }^{2}$ Institut für Festkörper- und Materialphysik, Technische Universität Dresden, D-01062 Dresden, Germany \\ ${ }^{3}$ Centro de Física de Materiales CFM-MPC and Centro Mixto CSIC-UPV/EHU, 20018 Donostia/San Sebastián, Basque Country, Spain \\ ${ }^{4}$ Tomsk State University, Lenina Av., 36, 634050 Tomsk, Russia \\ ${ }^{5}$ Swiss Light Source, Paul Scherrer Institute, CH-5232 Villigen-PSI, Switzerland \\ ${ }^{6}$ Kristall- und Materiallabor, Physikalisches Institut, Goethe-Universität Frankfurt, Max-von-Laue Strasse 1, \\ D-60438 Frankfurt am Main, Germany \\ ${ }^{7}$ IFW Dresden, Helmholtzstr. 20, D-01069 Dresden, Germany \\ ${ }^{8}$ European Synchrotron Radiation Facility, 71 Avenue des Martyrs, Grenoble, France \\ ${ }^{9}$ Saint Petersburg State University, Saint Petersburg 198504, Russia \\ ${ }^{10}$ Diamond Light Source, Harwell Campus, Didcot OX11 ODE, United Kingdom \\ ${ }^{11}$ Donostia International Physics Center (DIPC), 20080 Donostia/San Sebastián, Basque Country, Spain \\ ${ }^{12}$ Departamento de Fisica de Materiales UPV/EHU, 20080 Donostia/San Sebastián, Basque Country, Spain \\ ${ }^{13}$ Max-Planck-Institut für Chemische Physik fester Stoffe, D-01187 Dresden, Germany \\ ${ }^{14}$ IKERBASQUE, Basque Foundation for Science, 48011 Bilbao, Spain
}

(Received 13 July 2018; revised manuscript received 7 September 2018; published 27 September 2018)

\begin{abstract}
Strong spin-orbit coupling (SOC) in combination with a lack of inversion symmetry and exchange magnetic interaction proves to be a sophisticated instrument allowing efficient control of the spin orientation, energy and trajectories of two-dimensional (2D) electrons and holes trapped at surfaces or interfaces. Exploiting Kondo-related phenomena and crystal-electric-field effects at reduced dimensionalities opens new opportunities to handle their spin-dependent properties offering novel functionalities. We consider here a 2D Kondo lattice represented by a Si-Ir-Si-Yb (SISY) surface block of the heavy-fermion material $\mathrm{YbIr}_{2} \mathrm{Si}_{2}$. We show that the Kondo interaction with $4 f$ moments allows finely tuning the group velocities of the strongly spinpolarized carriers in 2D itinerant states of this noncentrosymmetric system. To unveil the peculiarities of this interaction, we used angle-resolved photoemission measurements complemented by first-principles calculations. We established that the strong SOC of the Ir atoms induces spin polarization of the 2D states in SISY block, while the 2D lattice of $\mathrm{Yb} 4 f$ moments acts as a source for coherent $f$ - $d$ interplay. The strong SOC and lack of inversion symmetry turn out to lead not only to the anticipated Rashba-like splitting of the 2D states, but also to spin splitting of the $4 f$ Kramers doublets. They couple temperature-dependently to the spin-polarized 2D states and thereby guide the properties of the latter.
\end{abstract}

DOI: 10.1103/PhysRevB.98.115157

\section{INTRODUCTION}

The realization of multiple different functionalities in a single material is a rapidly developing field of research, which is driven both by scientific curiosity and the prospect of novel applications. Great attention is paid to the capability to create highly spin-polarized two-dimensional electron states (2DESs) at surfaces or interfaces of functional materials and the manipulation of their properties [1-4]. Spin-orbit coupling (SOC), which pairs the spin degree of freedom with the orbital movement of electrons, is at the core of this research field and provides a rich source for novel physics and the design of new materials $[5,6]$.

\footnotetext{
*Corresponding author: denis.vyalikh@dipc.org
}

As is known, lack of inversion symmetry gives rise to the SOC-based Dresselhaus [7] and Rashba [8,9] phenomena, which manifest the momentum-dependent spin splitting of electronic bands. These effects open a path to implementation in spintronic applications $[5,10,11]$. In contrast to the material-specific Dresselhaus effect, the ability to tune the strength of the Rashba effect by an external gate voltage $[10,12]$ or magnetic field [13] is considered to be an inevitable ingredient to control the spin-polarized transport properties. Combination of SOC with ferroelectricity gives rise to a new class of recently discovered materials called ferroelectric Rashba semiconductors [14-16]. For these, the spin texture arises from the Rashba effect and can be controlled and switched via an external magnetic field [17].

At low dimensionalities, SOC drives many exotic phenomena like chiral spin textures in topological insulators [18], the appearance of strongly spin-polarized surface and interface 
states and quantum Hall phenomena [5]. On the other hand, combination of low dimensionality and strongly correlated electrons is known to result in unconventional electronic properties, which notably deviate from those characteristic of bulk materials [19-21]. For instance, dimensional tuning of quantum criticality in heavy-fermion systems can be achieved by suppressing the magnetic order and enhancing the effective electron mass in 2D heavy-fermion materials as compared to their 3D analogues [22]. Thus low-dimensional materials combining strong electron correlations with strong SOC are rather promising candidates for revealing new properties that can potentially provide novel [23] functionalities for technological applications, for example, in spintronic devices based on the spin-Hall effect in Kondo materials [24-27] or in noncentrosymmetric superconductors $[28,29]$.

As part of the development of SOC implementations, mechanisms allowing fine-grained control over the properties of spin-polarized electrons must be investigated. Therefore the class of rare-earth (RE) ternary compounds $\mathrm{RE} T_{2} \mathrm{Si}_{2}$ (RE and $T$ are rare-earth and transition metal atoms, correspondingly) of the $\mathrm{ThCr}_{2} \mathrm{Si}_{2}$ type structure [30] attracts considerable attention. Besides their unique bulk properties evolving from a delicate interplay of $4 f$ and $s p d$ electrons, these materials serve as toy models for studying exotic surface physics within the Si-T-Si-RE four layer of the Si-terminated surface [31-35].

In these systems, the SOC can be tuned by choice of suitable transition metal atoms. It gradually increases by exchanging Co for Rh [32,36] and further for Ir. The SOC-based phenomena will be rather weak for Co $3 d$ electrons, while they will be greatly enhanced for Ir $5 d$ orbitals [37]. As a competing ingredient, exchange magnetic interaction may be exploited by inserting elementary $4 f$ magnets like $\mathrm{Gd}$ as the RE component [33]. Because the orbital moment of the $\mathrm{Gd} 4 f$ shell vanishes $(\boldsymbol{L}=0)$ [38], the pure and large spin moment of Gd will be a strong and robust source of magnetic phenomena. Then, the Si-terminated surface of $\mathrm{GdIr}_{2} \mathrm{Si}_{2}$ seems a nice platform for studying the tunability of the spin-dependent properties of 2DESs in the presence and competition of the Rashba effect and exchange magnetic fields.

A rotation of the $4 f$ moments to a certain angle relative to the surface normal [34] may be achieved by coupling to a crystal electric field (CEF) [39-41]. To make use of notable CEF effects, a nonvanishing orbital moment $\boldsymbol{L}$ is needed, like for instance in Ho [34] or Dy [42]. Then, this option allows to implement an exchange magnetic field with different strength and orientation at the surface, which competes with the Rashba field and creates additional possibilities to manipulate the properties of the 2DESs [34].

Moreover, $\mathrm{RET}_{2} \mathrm{Si}_{2}$ materials offer the opportunity to explore how the effective masses and group velocities of highly spin-polarized 2DESs can be adjusted in the presence of strong electron correlations. For this purpose, we propose to exploit the interplay of the 2DESs with $4 f$ moments within a 2D Kondo lattice in the presence of strong spin-orbit coupling and a noncentrosymmetric environment. Such a scenario is realized at the $\mathrm{Si}$ surface within a $\mathrm{Si}-\mathrm{Ir}-\mathrm{Si}-\mathrm{Yb}$ (SISY) fourlayer block of the heavy-fermion material $\mathrm{YbIr}_{2} \mathrm{Si}_{2}$ [43-46], which is the actual object of our presented study.

The beauty of this block, which possesses the required noncentrosymmetric geometry, lies in the emergence of 2DESs that experience the combined influence of strong SOC and Kondo interaction due to the admixture of the $5 d$ states of Ir and the $4 f$ moments of $\mathrm{Yb}$. One of the striking results is the disclosure of momentum-dependent splitting of the $4 f$ states from the $\mathrm{Yb}$ subsurface layer due to the missing inversion symmetry. The interplay of these $4 f$ states with the itinerant spin-polarized 2DESs can be used as an instrument to finely manipulate the effective mass and group velocity of the latter. We propose and demonstrate that in general such a Si-T-Si-RE system may serve as a beautiful playground for studying the fundamental properties of $2 \mathrm{D}$ electrons. These systems can be nicely used as a veritable construction kit with spin-orbit, Kondo, crystal-electric field, and exchange magnetic interactions as building blocks. Combining them with one another gives the opportunity to design systems for different scenarios and to study the physics of 2DESs in the presence of these competing interactions.

\section{EXPERIMENT AND CALCULATION DETAILS}

\section{A. Experimental details}

The temperature-dependent ARPES experiments from $100 \mathrm{~K}$ down to $8 \mathrm{~K}$ were performed at the $\mathrm{I} 05$ beamline of the Diamond Light Source (DLS) [47], while the $1 \mathrm{~K}$ measurements were carried out at the "1-cubed ARPES" setup of the BESSY-II facility (HZB Berlin) [48]. High quality singlecrystalline samples of $\mathrm{YbIr}_{2} \mathrm{Si}_{2}$ with body-centred structure of I-type [Fig. 1(a)] were grown using a high-temperature indium-flux method [46]. The silicon-terminated surface [Fig. 1(c)] has been identified by the presence of a pronounced surface state at the $\bar{M}$ point [31-35], labeled as $\alpha$ band, as well as by a surface core-level shift in the $\mathrm{Yb} 4 f$ emission [49].

\section{B. Theoretical modelling}

$A b$ initio calculations were performed with the projector augmented-wave method [50] (VASP code [51,52]) and generalized gradient approximation to the exchange-correlation potential [53]. The Hamiltonian contained scalar-relativistic corrections and spin-orbit coupling was taken into account by a second-variation procedure [54]. We set the energy cutoff for the plane-wave expansion of wave functions to $253 \mathrm{eV}$ and sampled the two-dimensional (three-dimensional) Brillouin zone with a $18 \times 18 \times 1(18 \times 18 \times 7) k$-point grid .

Experimental lattice constants were used in the calculations, which for our $\mathrm{YbIr}_{2} \mathrm{Si}_{2}$ samples were measured to be $a=4.0345 \AA$ and $c=9.8282 \AA$ [46]. The Si-terminated (001) surface was simulated by a symmetric slab with 19 atomic layers of $P 4 / \mathrm{nmm}$ symmetry (space group 129). Our analysis performed for slabs of different symmetry $(99,123$, or 129 space group) and thickness (up to 32 layers) allowed to conclude that this choice is optimal: 19 layers turned out to be both necessary and sufficient to describe the essential features of the Si-terminated $\mathrm{YbIr}_{2} \mathrm{Si}_{2}$ surface electronic structure. Due to the inversion symmetry of the 19-layer slab, the socalled hidden spin polarization [55] was calculated as in-plane spin projection onto the upper half of the slab. The four utmost atomic layers comprising a surface SISY block were allowed to relax until the forces acting on atoms were less than 0.01 $\mathrm{eV} / \AA$, while the rest of the atoms were fixed at their relaxed 
(a)

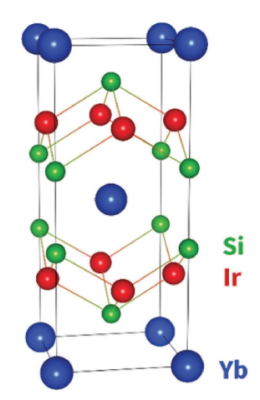

(b)

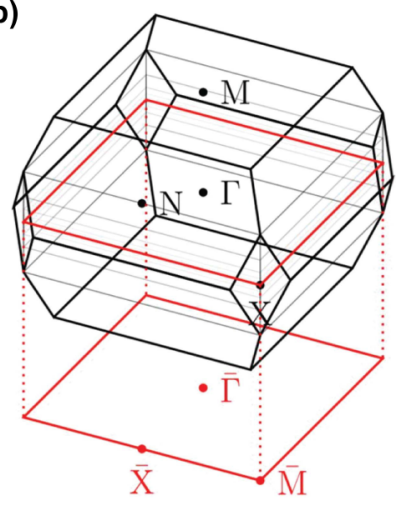

(c)

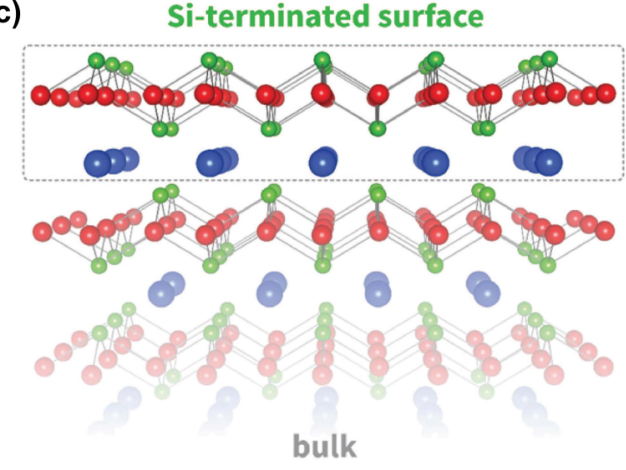

FIG. 1. Tetragonal crystal structure of $\mathrm{YbIr}_{2} \mathrm{Si}_{2}$. (a) Bulk unit cell. The $\mathrm{Yb}$ atomic layers are separated from each other by the tightly bonded $\mathrm{Si}$-Ir-Si trilayer blocks thus the cleavage plane will be between $\mathrm{Si}$ and $\mathrm{Yb}$ layers. (b) Evolution of the Brillouin zone from bulk to surface symmetry. The black zone corresponds to $I 4 / \mathrm{mmm}$, grey to $P 4 / \mathrm{mmm}$, and red to $P 4 \mathrm{~mm}$ and $P 4 / \mathrm{nmm}$. (c) Si-terminated surface of $\mathrm{YbIr}_{2} \mathrm{Si}_{2}$. The surface related $\mathrm{Si}-\mathrm{Ir}-\mathrm{Si}-\mathrm{Yb}$ (SISY) block is surrounded by a dotted line.

bulk positions. During site relaxation the $\mathrm{Yb} 4 f$ electrons were placed in the core to speed up the process. Our test calculations performed for the bulk revealed that the effect of the $4 f$ electrons on the atomic relaxations is marginal. For the relaxed slab, the electronic structure calculations were performed with the $\mathrm{Yb} 4 f$ electrons in the valence band.

\section{RESULTS AND DISCUSSION}

In Fig. 2(a), we present a low-temperature ARPES overview spectrum taken from a Si-terminated surface of a $\mathrm{YbIr}_{2} \mathrm{Si}_{2}$ crystal along the $\bar{M}-\bar{X}$ direction of the surface Brillouin zone (SBZ), see Figs. 1(b) and 1(c). Exploring this image, we can clearly observe a few strongly dispersing bands stemming from itinerant electrons along with a fine, well-defined, and nearly dispersionless spectral pattern close to the Fermi level $\left(E_{F}\right)$. The latter originates from the ${ }^{2} F_{7 / 2}$ manifold of the $\mathrm{Yb} 4 f$ states. It shows a narrow structure of finely split states caused by the CEF $[39,40,56]$. The resulting $\mathrm{Yb} 4 f$ Kramers doublets seen as individual flat bands running throughout the presented $k$-space region are tightly packed within a narrow binding energy (BE) range of the order of $70 \mathrm{meV}$

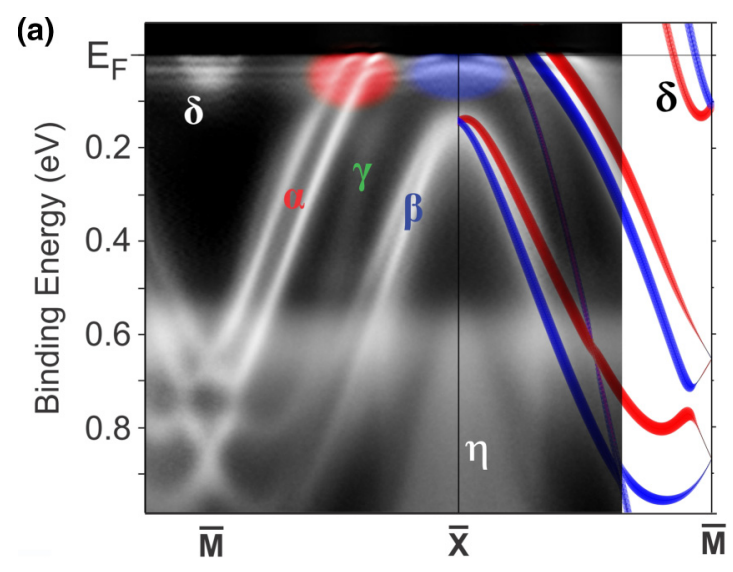

(b)

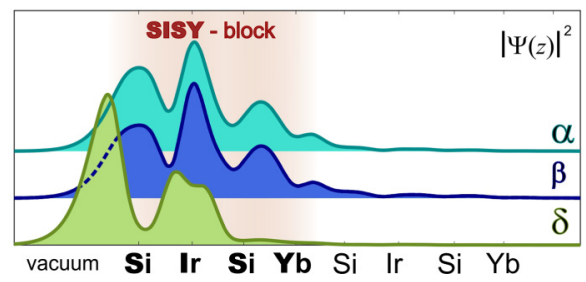

FIG. 2. Spectral pattern reflecting itinerant and $4 f$ electron bands. (a) ARPES data taken from a Si-terminated $\mathrm{YbIr}_{2} \mathrm{Si}_{2}$ crystal at a temperature of $1 \mathrm{~K}$ along the $\bar{M}-\bar{X}$ direction and superimposed with itinerant bands calculated by DFT. The spin polarization of the calculated bands is highlighted in red (in-plane positive $\sigma_{x}$ component) and blue (in-plane negative $\sigma_{x}$ component). The observable regions of the interplay between $f$ and $s p d$ states at $E_{F}$ are highlighted with red and blue patches. (b) Electron density distribution (integrated over the $a b$ plane) of the 2DESs residing in the surface SISY block as calculated near the $\bar{M}$ point (at the $k$ points $\approx 1 / 4$ th of the $\bar{M}-\bar{X}$ distance for the $\alpha$ and $\beta$ states and 1/16th of $\bar{M}-\bar{X}$ for the $\delta$ states, respectively).

In spite of their strong localization at the $\mathrm{Yb}$ sites, the $4 f$ states must obey the same symmetry rules imposed by the crystal as the broad itinerant bands. The reason they are resolved so clearly and are pinned to the Fermi level $E_{F}$ can be readily explained by the mixed valency of the considered Kondo material. In such a system the $4 f^{n}$ and $4 f^{n-1}$ electron configurations are mixed in the ground state. When an $f$ electron is photoexcited, the $4 f^{n-1}$ component of the initial state is reproduced by the $4 f^{n-1}$ component of the final state due to the excitation of the $4 f^{n}$ configuration [57]. Both initial and final state configurations lie very close in energy to each other and therefore appear at $E_{F}$ in the ARPES experiment. The long lifetime of these CEF-split $4 f$ states results in spectral features that are extremely narrow. This allows to scrutinize their $k$-dependent properties and the peculiarities of interplay with the widely dispersing itinerant bands [39]. When these two electronic subsystems meet each other, the mutual interaction is reflected in characteristic splittings and gaps, which are the subject of our attention. These regions of $k$ space at $E_{F}$ are highlighted in red- and blue-colored patches in Fig. 2(a).

Let us turn first to the itinerant bands, which are marked as $\alpha, \beta, \gamma, \delta$, and $\eta$. Looking closely, we can see that, for example, the bands $\alpha$ and $\beta$ reveal a pronounced splitting. 
Note that $\mathrm{YbIr}_{2} \mathrm{Si}_{2}$ at the considered temperature of $1 \mathrm{~K}$ is in the paramagnetic Kondo regime [43,46]. Therefore these splittings cannot be explained by exchange magnetic phenomena. To shed further light on this issue, we have performed band structure calculations, where the system was modeled as a slab of 19 atomic layers terminated with a SISY block on both sides (see the Experiment and Calculation details section). The $4 f$ orbitals were treated as frozen core states, allowing to model the itinerant bands dispersion in absence of $f-d$ interaction. The theoretically derived bands are superimposed on the ARPES data in Fig. 2(a). Both calculated and experimental bands are in very good agreement. All the dispersive bands (i.e., apart from the $4 f$ states, which were excluded from the variational basis set) and their splittings, which were found to be due to the Rashba effect, were nicely reproduced. The calculations demonstrate that the $\alpha, \beta$, and $\delta$ states seen in the ARPES experiment are surface states, while the bands $\gamma$ and $\eta$ (not shown) are surface-projected bulk states. It is worth noting that the $f-d$ hybrids clearly seen in the ARPES pattern at $E_{F}$, which also includes the $\gamma$ and $\eta$ bands, constitute the well-known jungle gym and doughnut Fermi surface (FS) sheets [58,59], respectively, which will be discussed later.

From an analysis of the orbital contributions within the individual atomic layers of the slab, our calculations suggest that the $\alpha, \beta$, and $\delta$ bands belong exclusively to the SISY block. As clearly seen from the density profiles $|\Psi|^{2}$ for the discussed states at $k$ in the vicinity of $\bar{M}$, see Fig. 2(b), the bands $\alpha$ and $\beta$ are located within a full SISY block overlapping simultaneously with the Ir and $\mathrm{Yb}$ layers. The band $\delta$ on the other hand is localized mainly within the outermost Si-Ir double layer, implying its coupling with the $4 f$ states of the Yb layer will be weak. Thus, the bands $\alpha$ and $\beta$ seem to be the most interesting candidates for 2DESs, where firstly strong spin polarization apparently occurs, and secondly in particular band $\alpha$ crosses and couples with the $4 f$ bands at the $E_{F}$. Note that the ARPES measurements were performed up to $100 \mathrm{~K}$ and revealed that the discussed SOC splittings remain unchanged up to the highest applied temperature. The surface states show the Rashba-like spin splitting almost over the whole $k$-interval considered, with the exception of the close vicinity of the $\bar{M}$ point for the $\alpha$ and $\beta$ bands [see Fig. 2(a)]. Approaching the corner of the SBZ, these bands start to interact and acquire a spin structure with an almost compensated spin, although still perpendicularly locked to momentum in the $\bar{X}-\bar{M}$ direction as expected for the Rashba effect [5].

To gain further insight into the peculiarities of interaction between $\mathrm{Yb} 4 f \mathrm{CEF}$ states and surface-electron states, we consider a high-resolution close-up ARPES map taken at $1 \mathrm{~K}$ and shown in Fig. 3(a). The normalized ARPES data reveal a bunch of closely packed $4 f$ bands. These flat bands exhibit a rather complex dispersion in particular when they pass through the $\alpha$ band and when they approach the $\bar{X}$ point. We can distinguish that one $4 f$ state appears right below the $E_{F}$ bending upwards and crossing it making a "neck" around the $\bar{X}$ point, marked by blue arrows in Fig. 3(a). It is also seen that another $4 f$ band, lying slightly deeper in $\mathrm{BE}$, shows a similar holelike shape of its dispersion near the $\bar{X}$ point. Its top is about $20 \mathrm{meV}$ below $E_{F}$. The similar dispersion implies
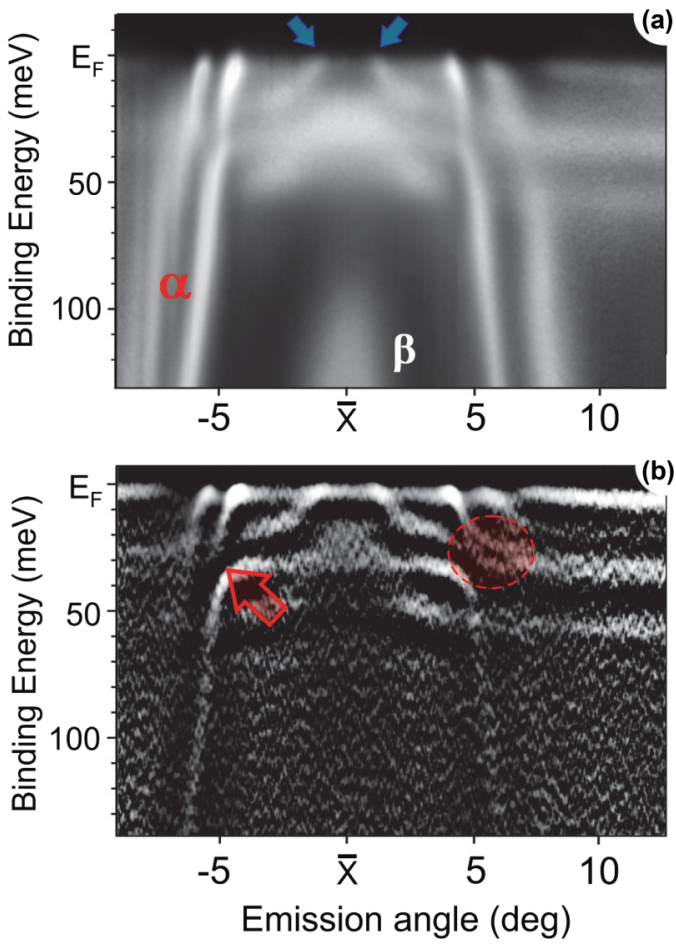

FIG. 3. Close-up ARPES maps disclosing the interplay of spinpolarized $2 \mathrm{D}$ and $4 f$ states. Normalized raw data in (a) obtained at $T=1 \mathrm{~K}$ and $h v=45 \mathrm{eV}$ along the $\bar{X}-\bar{M}$ direction in the SBZ. Blue arrows indicate the "neck" of doughnut FS. (b) Second derivative applied along the energy distribution curves (EDC) of the map in (a). This allows to disclose the fine spectral details revealing a "looplike" splitting of a $4 f$ CEF Kramers doublet marked by the red circle. It helps also to better visualize the hybridization (avoided-crossing) gaps as the one marked by a red arrow.

similar symmetry properties of these bands, which will be discussed later. It is worth noting that the "neck" feature is a signature of the fundamental doughnut Fermi surface of $\mathrm{YbT}_{2} \mathrm{Si}_{2}$ systems ( $T=\mathrm{Co}, \mathrm{Rh}, \mathrm{Ir}$ ) [32,58,59]. It can be seen in ARPES experiments as a signature of the hybridization of $4 f$ states of the subsurface $\mathrm{Yb}$ atoms (the fourth layer of the SISY block) with the projected bulk states. Further below, this interplay will be discussed in more detail by considering temperature-dependent APRES data.

Let us now precisely look at the crossing region of the $4 \mathrm{fs}$ with the spin-polarized $\alpha$ band. The signature of interaction is also seen there, however, a bit less pronounced but the avoided crossing gaps due to mutual interplay can be still distinguished. The visibility of these gaps can be enhanced by the second derivative applied to the energy distribution curves as shown in Fig. 3(b). Obviously, the mentioned gapped feature reflecting hybridization of the $4 f \mathrm{~s}$ with the $\alpha$ band can be better seen now as is marked by a red arrow.

We consider now the symmetry of the $4 f \mathrm{CEF}$ states. The ground state of the $4 f^{13}$ configuration of $\mathrm{Yb}^{3+}$ is a ${ }^{2} F_{7 / 2}$ multiplet with $J=7 / 2$. In the tetragonal crystal field at the $\mathrm{Yb}$ site of the bulk material ( $I 4 / \mathrm{mmm}$, space group 139) this eightfold degenerate state splits into four Kramers doublets corresponding to the irreducible representations $\Gamma_{6}^{-}$and $\Gamma_{7}^{-}$ [56] at the BZ center. The missing inversion symmetry at the 

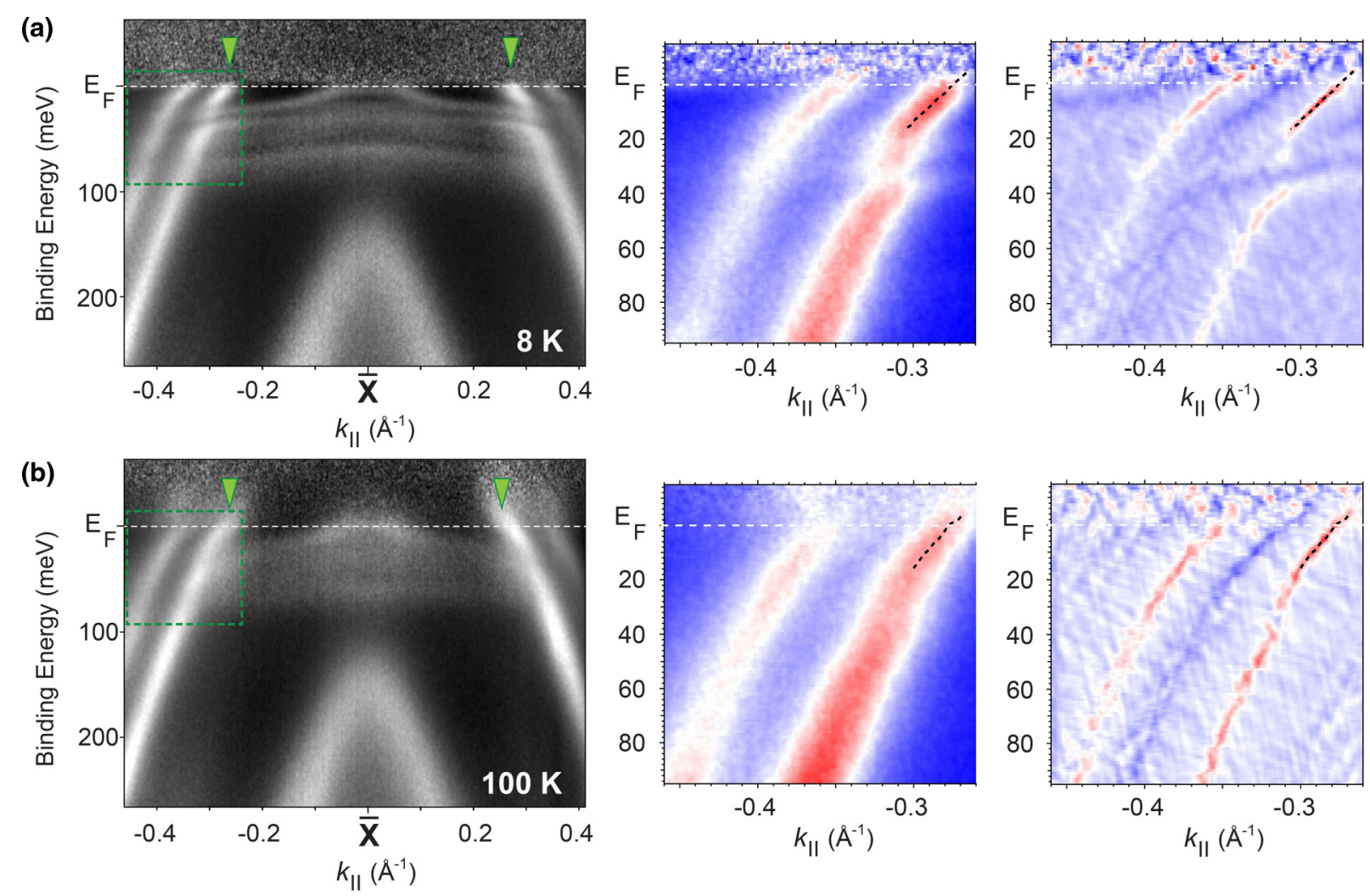

(c)
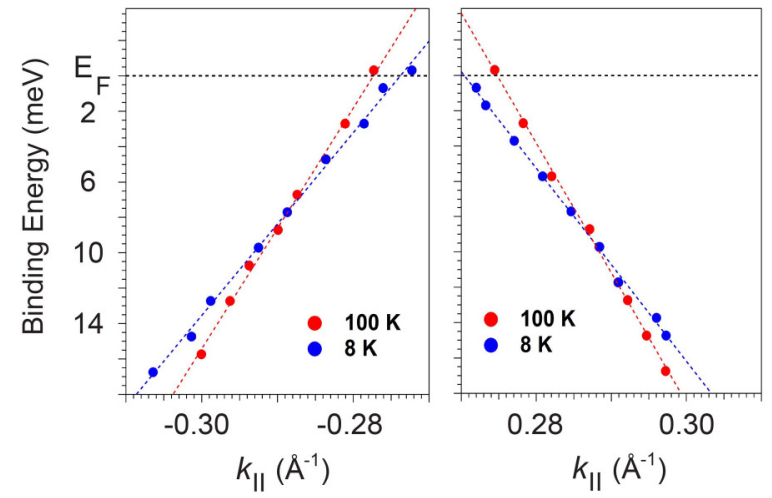

(d)

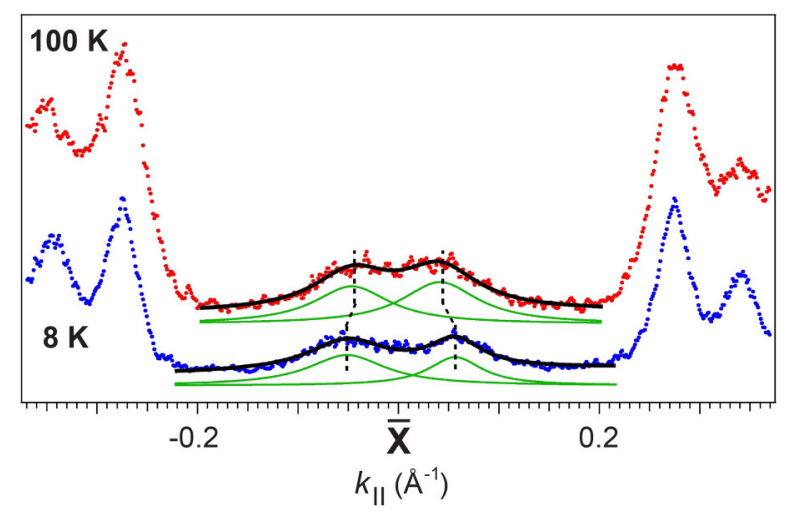

FIG. 4. Temperature-dependent properties of the $f-d$ interaction. ARPES-derived spectral pattern reflecting the topology of the hybrid $f-d$ states obtained at 8 (a) and $100 \mathrm{~K}$ (b). The MDC-normalized data magnified within the dashed green rectangle in the left panels in (a) and (b) are depicted in the middle panels together with the applied curvature procedure in the right panels. The derived dispersion for the $\alpha$ bands near $E_{F}$ (marked by green triangles) experiencing renormalization due to spin-dependent interplay with the $4 f \mathrm{~s}$ is shown in (c) for $8 \mathrm{~K}$ and $100 \mathrm{~K}$ on both sides of the $\bar{X}$ point. (d) MDC curves at $E_{F}$ extracted for 8 and $100 \mathrm{~K}$ demonstrating the narrowing of the "neck" of the $4 f$-derived doughnut $\mathrm{FS}$ at higher temperature.

surface ( $P 4 m m$, space group 99$)$ and electronic dispersion away from the $\bar{\Gamma}$ point of the SBZ both further reduce the symmetries of the wave function $[60,61]$. At the $\bar{M}$ point, the full surface symmetry remains and there are four doublets with the symmetries $\bar{M}_{6}$ and $\bar{M}_{7}$. At $\bar{X}$, four doublets (all of the symmetry $\bar{X}_{5}$ ) are also expected. According to the compatibility relations [61], along the path $\bar{Y}$ between the two points, however, the remaining degeneracy of the doublets lifts: each doublet is split into two levels of the symmetry $\bar{Y}_{3}$ and $\bar{Y}_{4}$ as well as the surface states $(\alpha, \beta$, and $\delta)$ residing in the SISY block. By itself, the potential asymmetry at the Yb site is likely not strong enough to effectively split the respective $f$ bands as it is relatively far from the surface taking into account the small $4 f$ orbital extent. However, an interaction between the $4 f$ 's and the strongly spin-split 2DESs can induce a spin split in the $4 f$ levels proportional to the interaction strength. This would result in a "loop" or "hysteresis"-like dispersion due to the avoided crossing of levels of the same symmetry $\left(\overline{\mathrm{Y}}_{3}\right.$ or $\left.\overline{\mathrm{Y}}_{4}\right)$. Apparently, this feature was extracted in Fig. 3(b) at the crossing between the most intense CEF level and the strongly split surface state highlighted by a red circle. Summarizing the results of the analysis of Fig. 3 we may conclude that symmetry allows all four Kramers doublets to be nondegenerate beyond the time-reversal invariant momenta 
and to couple spin-selectively to the spin-polarized $\alpha$ band. For the first time, we thus observe experimentally the spin dependent coupling that, as we simulate below by means of $a b$ initio calculations, should results in a Rashba type indirectly induced spin-polarization of the Kramers doublets.

Further, we have performed temperature-dependent ARPES measurements of the spectral structure along the $\bar{M}-\bar{X}-\bar{M}$ direction. In Fig. 4, we have summarized the obtained results and their analysis for 8 and $100 \mathrm{~K}$. First, we focus on the evolution of the spectral pattern reflecting the interplay of the SOC split $\alpha$-band with the $4 f$ states near $E_{F}$. In Figs. 4(a) and 4(b), we show the raw ARPES band maps along with enhanced dispersions extracted by the curvature-plot procedure [62]. By fitting the dispersive peaks in the momentum distribution curves (MDC) of the bands marked by green triangles, we were able to extract the changes of their slopes as a function of temperature. The slopes reflecting the electron velocity are shown in Fig. 4(c).

As seen in the figure, at the temperature of $100 \mathrm{~K}$, the corresponding spin-polarized electrons become slightly faster: the group velocity increases from $(0.79 \pm 0.02) \times 10^{5} \mathrm{~m} / \mathrm{s}$ at $8 \mathrm{~K}$ up to $(1.03 \pm 0.02) \times 10^{5} \mathrm{~m} / \mathrm{s}$ at $100 \mathrm{~K}$ as found for the $\alpha$ band on the left side of the $\bar{X}$ point and from $(0.83 \pm 0.02) \times 10^{5} \mathrm{~m} / \mathrm{s}$ at $8 \mathrm{~K}$ to $(1.12 \pm 0.02) \times 10^{5} \mathrm{~m} / \mathrm{s}$ at $100 \mathrm{~K}$ on the right side.

The second remarkable aspect becomes evident in the analysis of the MDCs at the Fermi level, which are shown in Fig. 4(d). We clearly see that the "neck" of the $4 f$-derived doughnut Fermi surface sheet mentioned above obviously becomes narrower with increasing temperature explicitly indicating the fundamental process of transition from a large to a small (holelike) Fermi surface in a Kondo lattice [58]. The issue of the transition from small to large FS is presently hotly discussed [63-65]. Most recent papers on this subject suggest that $f-d$ hybridization persists at temperatures much higher than the Kondo temperature $[64,65]$. They suggest that the transition from the small to the large Fermi surface is a smooth process, however, it has not yet been experimentally established for $\mathrm{YbT}_{2} \mathrm{Si}_{2}$ Kondo lattices. The essential point here that the SISY of $\mathrm{YbIr}_{2} \mathrm{Si}_{2}$ still remains in the Kondo regime at $100 \mathrm{~K}$ with somewhat softer $f-d$ interplay than that at $8 \mathrm{~K}$. Thus, these observations point out that the group velocity of the 2D spin-polarized electrons at $E_{F}$, which hybridize with the $\mathrm{Yb} 4 f$ moments, is gently modified by the Kondo interaction. At higher temperature, when the $4 f$ electrons behave as local moments, the $2 \mathrm{D}$ electrons remain fast. At low temperature when the system enters the Kondo regime, the velocity of the spin-polarized 2DESs is reduced due to the coherent Kondo effect. Beside, the observation of the narrowing neck at the $\bar{X}$ point with increasing temperature on its own is a remarkable experimental result, which manifests direct evidence by ARPES of the transition process from large to a small Fermi surface in $\mathrm{YbIr}_{2} \mathrm{Si}_{2}$, in particular, and for the first time for an $\mathrm{Yb}$-based Kondo lattice, in general.

To estimate the velocity of the considered $\alpha$ state without interaction with the $4 f$ electrons, we analyzed its dispersion and extracted the velocity at the BE range between 0.1 and $0.15 \mathrm{eV} \mathrm{BE}$, away from the region of its intersection with the $4 f$ states. The obtained value of $2.2 \times 10^{5} \mathrm{~m} / \mathrm{s}$ is more than twice higher than at low temperature. This implies that the
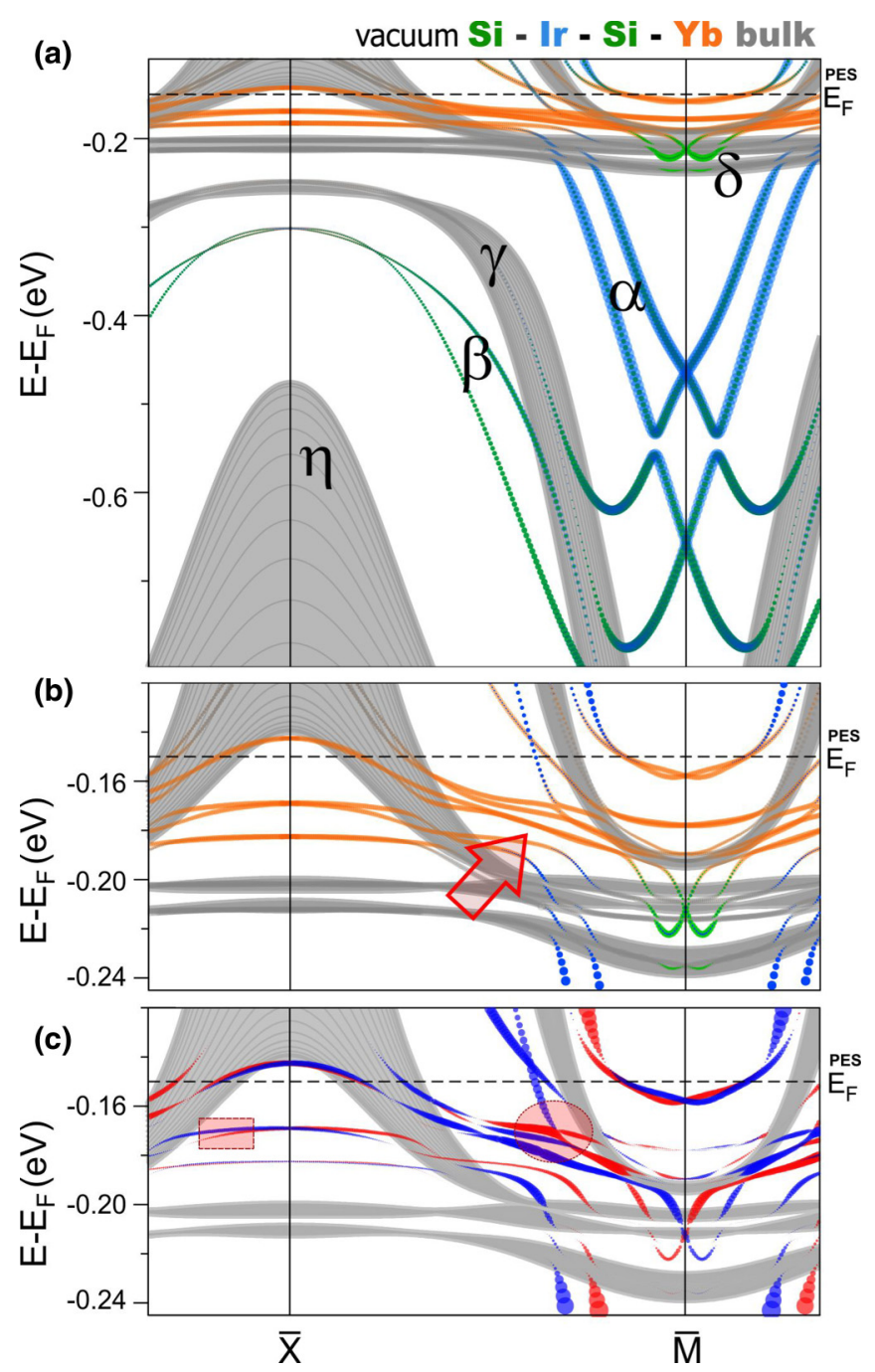

FIG. 5. All-itinerant DFT band structure and spin polarization of the Si-terminated surface of $\mathrm{YbIr}_{2} \mathrm{Si}_{2}$. (a) The fat bands of the 19-layer slab show the localization of the states on the atomic layers within the SISY block. Green, blue, and orange fat bands correspond to the Si-, Ir-, and Yb-atomic layer, respectively. The shaded areas cover the surface-projected bulk states of $\mathrm{YbIr}_{2} \mathrm{Si}_{2}$. The zero of the energy scale on the left side is the Fermi level within the DFT calculation. Best matching with experimental ARPES data would be achieved by shifting the Fermi level to the position marked on the right side with "PES $E_{F}$." (b) A magnified view of the $\mathrm{BE}$ range, where the $4 f$ bands interact with the 2DESs. (c) The spin polarization of the bands shown in the figure (b) with the enhanced contribution coming from the Ir and subsurface $\mathrm{Yb}$ atoms of the SISY block. The in-plane positive (negative) $\sigma_{x}$ component is highlighted in red (blue). The main features of the band dispersions related to the fine details of the ARPES spectral pattern are indicated by the arrow and the circle as in Fig. 3(b). The rectangle highlights the spin splitting of a $4 f$ band before it enters the bulk continuum.

2D electrons can be notably slowed down through interaction with the $\mathrm{Yb} 4 f$ states close to the Fermi level by lowering the temperature by one order of magnitude. Our results suggest that $f$ - $d$ interaction is a capable instrument to finely tune the group velocities of itinerant states. The new point here is the strong spin-orbit coupling which makes these states 
highly spin-polarized and therefore moves these phenomena potentially into the field of interest for spintronics [26,27].

The revealed peculiarities of the $4 f$-2DES interplay in a 2D Kondo lattice realized in the SISY block of $\mathrm{YbIr}_{2} \mathrm{Si}_{2}$ call for a detailed theoretical description beyond the above considerations based solely on symmetry arguments. However, a state-of-the-art $a b$ initio method that would be a practicable theoretical tool to study strongly correlated systems of reduced dimensionality, is missing. Therefore, DFT can be a method of choice for the microscopic treatment of surface-related phenomena. The good performance of DFT, particularly, in the case of all-itinerant calculations of the bulk Fermi surface for the heavy-fermion material $\mathrm{YbRh}_{2} \mathrm{Si}_{2}$ [58] and their agreement with ARPES results $[59,63]$ justifies the use of the Kohn-Sham band structure with itinerant $\mathrm{Yb} 4 f$ electrons as a reasonable estimation.

In order to relate the main features of the acquired ARPES data to microscopically relevant band dispersions below the Fermi energy, we consider again a 19-layer slab (see Experiment and Calculation details section), but now treating the $\mathrm{Yb}$ $4 f$ electrons as itinerant. Apart from the surface- and bulkrelated features already present in the frozen-core calculation, here in the resulting band structure one finds quite narrow $f$ bands coming both from the bulk and from the subsurface $\mathrm{Yb}$ atoms, Fig. 5(a). The latter have a slightly different CEF environment as the bulk atoms due to the near-surface atomic configuration. This distinct ligand field manifests itself in a small upshift in BE by about $30 \mathrm{meV}$ of the subsurface $\mathrm{Yb}$ $4 f$ states with respect to the bulk $f$ states. It is worth noting that all the 2D states residing in the SISY block become surface resonances when they enter the projected bulk band continuum shaded in gray in Fig. 5(a).

In Fig. 5(b), a magnified view of the BE range that covers all the $4 f$ bands clearly shows the strong interplay of the 2DESs (highlighted blue and green) and the subsurface $\mathrm{Yb}$ $4 f$ states (highlighted orange). Along with the underlying projected bulk continuum (shaded in gray), which affects the dispersions of the SISY-block bands, the calculated spectral pattern resembles the one in Fig. 3. Note the good agreement of the $4 f$ bandwidth with experimental observations, which is clearly seen in the vicinity of the $\bar{M}$ point. At that, the absolute positions of the $\mathrm{Yb} 4 f$ states and the 2DESs on the energy scale with respect to the Fermi level differ within $\sim 0.2 \mathrm{eV}$ (see also Refs. [66,67]) from the experiment, where the former are closer to the Fermi level, while the latter are further from $E_{F}$, cf. Figs. 2(a) and 5(a). However, it seems plausible to relate a gap located right above the "transition" of the $\alpha$ band into the split $4 f$ bands of the subsurface $\mathrm{Yb}$ atoms in Fig. 5(b) with the avoided-crossing gap marked by the red arrow in Fig. 3(b). The "looplike" feature circled in
Fig. 3(b) can thus be referred to the splitting of the upper nearly dispersionless $4 f$ band induced by the interplay with the spin-polarized 2DESs as seen in Fig. 5(c). Importantly, the spin-resolved representation of the SISY-block bands reveals a complex and intricate spin polarization of the $4 f$ bands. Besides spin, $4 f$ electrons carry a large orbital angular momentum, which results in the SOC-induced splitting of the band that is nonlinear in the momentum $k$ and accompanied by a change of spin polarization within a branch of the split band.

\section{CONCLUSION}

In summary, we have demonstrated and explored the new opportunities, which are promising for control of spinpolarized two-dimensional electron states by combining strong spin-orbit coupling with Kondo physics at low dimensionality. As an example we studied the two-dimensional Kondo lattice realized in the Si-Ir-Si-Yb surface block of the heavy-fermion material $\mathrm{YbIr}_{2} \mathrm{Si}_{2}$. In conjunction with the intrinsic lack of inversion symmetry, strong SOC leads to splitting and consequent spin polarization not only of the itinerant bands, but also of the $4 f$ states of near-surface rare-earth atoms, which is reflected in fine experimentally resolved splittings of the $f$-derived heavy bands. At elevated temperature, the strongly spin-polarized 2D states are highly mobile, while at lower temperature they start to coherently couple with the $4 f$ moments leading to a reduction of their group velocity. The discovered splitting of heavy electron bands opens new degrees of freedom for novel applications involving spin-polarized $4 f$ electrons in elaborated nanostructures.

\section{ACKNOWLEDGMENTS}

This work was supported by the German Research Foundation (DFG; Grants No. GRK1621, No. KR3831/5-1, No. GE 602/4-1 Fermi Nest and No. SFB1143) as well as Research Grant No. 15.61.202.2015 of Saint-Petersburg State University. We also acknowledge the funding by the Basque Departamento de Educacion, UPV/EHU (Grant No. IT-75613), the Spanish Ministry of Economy, Industry and Competitiveness MINEICO (Grants No. FIS2016-75862-P and No. FIS2016-76617-P), and Tomsk State University competitiveness improvement programme (project No. 8.1.01.2017). The calculations were performed in Donostia International Physics Center and in the Research park of the St. Petersburg State University Computing Center (http://cc.spbu.ru). We acknowledge Diamond Light Source for time on Beamline I05 under proposal SI14811.
[1] A. Manchon, H. C. Koo, J. Nitta, S. M. Frolov, and R. A. Duine, New perspectives for Rashba spin-orbit coupling, Nat. Mater. 14, 871 (2015).

[2] E. E. Krasovskii, Spin-orbit coupling at surfaces and 2D materials, J. Phys.: Condens. Matter 27, 493001 (2015).
[3] Wei Han, Perspectives for spintronics in 2D materials, APL Mater. 4, 032401 (2016).

[4] L. Fang, J. Im, W. DeGottardi, Y. Jia, A. Glatz, K. A. Matveev, W.-K. Kwok, G. W. Crabtree, and M. G. Kanatzidis, Large spinorbit coupling and helical spin textures in 2D heterostructure $\left[\mathrm{Pb}_{2} \mathrm{BiS}_{3}\right]\left[\mathrm{AuTe}_{2}\right]$, Sci. Rep. 6, 35313 (2016). 
[5] A. Soumyanarayanan, N. Reyren, A. Fert, and C. Panagopoulos, Emergent phenomena induced by spin-orbit coupling at surfaces and interfaces, Nature (London) 539, 509 (2016).

[6] J.-C. Rojas-Sánchez, S. Oyarzún, Y. Fu, A. Marty, C. Vergnaud, S. Gambarelli, L. Vila, M. Jamet, Y. Ohtsubo, A. Taleb-Ibrahimi, P. Le Fèvre, F. Bertran, N. Reyren, J.-M. George, and A. Fert, Spin to Charge Conversion at Room Temperature by Spin Pumping into a New Yype of Topological Insulator: $\alpha$-Sn Films, Phys. Rev. Lett. 116, 096602 (2016).

[7] G. Dresselhaus, Spin-orbit coupling effects in zinc blende structures, Phys. Rev. 100, 580 (1955).

[8] E. I. Rashba and V. I. Sheka, Symmetry of energy bands in crystals of wurtzite type ii. symmetry of bands with spin-orbit interaction included, Fiz. Tverd. Tela: Collected Papers 2, 62 (1959)

[9] Yu. A. Bychkov and E. I. Rashba, Properties of a 2D electron gas with lifted spectral degeneracy, JETP Lett. 39, 78 (1984).

[10] E. Lesne, Y. Fu, S. Oyarzun, J. C. Rojas-Sánchez, D. C. Vaz, H. Naganuma, G. Sicoli, J. P. Attané, M. Jamet, E. Jacquet, J. M. George, A. Barthélémy, H. Jaffrès, A. Fert, M. Bibes, and L. Vila, Highly efficient and tunable spin-to-charge conversion through Rashba coupling at oxide interfaces, Nat. Mater. 15, 1261 (2016).

[11] I. A. Nechaev and E. E. Krasovskii, Spin filtering via resonant reflection of relativistic surface states, Phys. Rev. B 97, 041407(R) (2018).

[12] A. D. Caviglia, M. Gabay, S. Gariglio, N. Reyren, C. Cancellieri, and J.-M. Triscone, Tunable Rashba Spin-Orbit Interaction at Oxide Interfaces, Phys. Rev. Lett. 104, 126803 (2010)

[13] C. Carbone, P. Moras, P. M. Sheverdyaeva, D. Pacilé, M. Papagno, L. Ferrari, D. Topwal, E. Vescovo, G. Bihlmayer, F. Freimuth, Y. Mokrousov, and S. Blügel, Asymmetric band gaps in a Rashba film system, Phys. Rev. B 93, 125409 (2016).

[14] S. Picozzi, Ferroelectric Rashba semiconductors as a novel class of multifunctional materials, Frontiers in Physics 2, 10 (2014).

[15] M. Liebmann, C. Rinaldi, D. D. Sante, J. Kellner, C. Pauly, R. N. Wang, J. E. Boschker, A. Giussani, S. Bertoli, M. Cantoni, L. Baldrati, M. Asa, I. Vobornik, G. Panaccione, D. Marchenko, J. Sánchez-Barriga, O. Rader, R. Calarco, S. Picozzi, R. Bertacco, and M. Morgenstern, Giant Rashba-type spin splitting in ferroelectric GeTe(111), Adv. Mater. 28, 560 (2015).

[16] H. J. Elmers, R. Wallauer, M. Liebmann, J. Kellner, M. Morgenstern, R. N. Wang, J. E. Boschker, R. Calarco, J. SánchezBarriga, O. Rader, D. Kutnyakhov, S. V. Chernov, K. Medjanik, C. Tusche, M. Ellguth, H. Volfova, St. Borek, J. Braun, J. Minár, H. Ebert, and G. Schönhense, Spin mapping of surface and bulk Rashba states in ferroelectric $\alpha$-GeTe(111) films, Phys. Rev. B 94, 201403 (2016).

[17] J. Krempaský, S. Muff, F. Bisti, M. Fanciulli, H. Volfová, A. P. Weber, N. Pilet, P. Warnicke, H. Ebert, J. Braun, F. Bertran, V. V. Volobuev, J. Minár, G. Springholz, J. H. Dil, and V. N. Strocov, Entanglement and manipulation of the magnetic and spin-orbit order in multiferroic Rashba semiconductors, Nat. Commun. 7, 13071 (2016).

[18] M. Z. Hasan and C. L. Kane, Colloquium: Topological insulators, Rev. Mod. Phys. 82, 3045 (2010).

[19] M. Shimozawa, Swee K. Goh, T. Shibauchi, and Y. Matsuda, From Kondo Lattices to Kondo Superlattices, Rep. Prog. Phys. 79, 074503 (2016).
[20] D. J. Groenendijk, C. Autieri, J. Girovsky, M. C. MartinezVelarte, N. Manca, G. Mattoni, A. M. R. V. L. Monteiro, N. Gauquelin, J. Verbeeck, A.F. Otte, M. Gabay, S. Picozzi, and A. D. Caviglia, Spin-Orbit Semimetal $\mathrm{SrIrO}_{3}$ in the TwoDimensional Limit, Phys. Rev. Lett. 119, 256403 (2017).

[21] P. Schütz, D. Di Sante, L. Dudy, J. Gabel, M. Stübinger, M. Kamp, Y. Huang, M. Capone, M.-A. Husanu, V. N. Strocov, G. Sangiovanni, M. Sing, and R. Claessen, Dimensionality-Driven Metal-Insulator Transition in Spin-Orbit-Coupled $\mathrm{SrIrO}_{3}$, Phys. Rev. Lett. 119, 256404 (2017).

[22] H. Shishido, T. Shibauchi, K. Yasu, T. Kato, H. Kontani, T. Terashima, and Y. Matsuda, Tuning the dimensionality of the heavy fermion compound $\mathrm{CeIn}_{3}$, Science 327, 980 (2010).

[23] G. Cao, J. Terzic, H. D. Zhao, H. Zheng, L. E. De Long, and P. S. Riseborough, Electrical Control of Structural and Physical Properties via Strong Spin-Orbit Interactions in $\mathrm{Sr}_{2} \mathrm{IrO}_{4}$, Phys. Rev. Lett. 120, 017201 (2018).

[24] T. Tanaka and H. Kontani, Intrinsic spin and orbital Hall effects in heavy-fermion systems, Phys. Rev. B 81, 224401 (2010).

[25] S. Nair, S. Wirth, S. Friedemann, F. Steglich, Q. Si, and A. J. Schofield, Hall effect in heavy fermion metals, Adv. Phys. 61, 583 (2012).

[26] T. Yoshida, R. Peters, and N. Kawakami, Restoration of topological properties at finite temperatures in a heavy-fermion system, Phys. Rev. B 93, 045138 (2016).

[27] N. Reynolds, P. Jadaun, J. T. Heron, C. L. Jermain, J. Gibbons, R. Collette, R. A. Buhrman, D. G. Schlom, and D. C. Ralph, Spin Hall torques generated by rare-earth thin films, Phys. Rev. B 95, 064412 (2017).

[28] M. Smidman, M. B. Salamon, H. Q. Yuan, and D. F. Agterberg, Superconductivity and spin-orbit coupling in noncentrosymmetric materials: A review, Rep. Prog. Phys. 80, 036501 (2017).

[29] P. K. Rout, E. Maniv, and Y. Dagan, Link between the Superconducting Dome and Spin-Orbit Interaction in the (111) $\mathrm{LaAlO}_{3} / \mathrm{SrTiO}_{3}$ Interface, Phys. Rev. Lett. 119, 237002 (2017).

[30] I. Felner and I. Nowik, Itinerant and local magnetism, superconductivity and mixed valency phenomena in $R M_{2} \mathrm{Si}_{2},(R=$ rare earth, $M=\mathrm{Rh}, \mathrm{Ru}$ ), J. Phys. Chem. Solids 45, 419 (1984).

[31] A. Chikina, M. Höppner, S. Seiro, K. Kummer, S. Danzenbächer, S. Patil, A. Generalov, M. Güttler, Yu. Kucherenko, E. V. Chulkov, Yu. M. Koroteev, K. Köpernik, C. Geibel, M. Shi, M. Radovic, C. Laubschat, and D. V. Vyalikh, Strong ferromagnetism at the surface of an antiferromagnet caused by buried magnetic moments, Nat. Commun. 5, 3171 (2014).

[32] M. Güttler, K. Kummer, S. Patil, M. Höppner, A. Hannaske, S. Danzenbächer, M. Shi, M. Radovic, E. Rienks, C. Laubschat, C. Geibel, and D. V. Vyalikh, Tracing the localization of $4 f$ electrons: Angle-resolved photoemission on $\mathrm{YbCo}_{2} \mathrm{Si}_{2}$, the stable trivalent counterpart of the heavy-fermion $\mathrm{YbRh}_{2} \mathrm{Si}_{2}$, Phys. Rev. B 90, 195138 (2014).

[33] M. Güttler, A. Generalov, M. M. Otrokov, K. Kummer, K. Kliemt, A. Fedorov, A. Chikina, S. Danzenbächer, S. Schulz, E. V. Chulkov, Yu. M. Koroteev, N. Caroca-Canales, M. Shi, M. Radovic, C. Geibel, C. Laubschat, P. Dudin, T. K. Kim, M. Hoesch, C. Krellner, and D. V. Vyalikh, Robust and tunable itinerant ferromagnetism at the silicon surface of the antiferromagnet $\mathrm{GdRh}_{2} \mathrm{Si}_{2}$, Sci. Rep. 6, 24254 (2016).

[34] A. Generalov, Mikhail M. Otrokov, A. Chikina, K. Kliemt, K. Kummer, M. Höppner, M. Güttler, S. Seiro, A. Fedorov, 
S. Schulz, S. Danzenbächer, Evgueni V. Chulkov, C. Geibel, C. Laubschat, P. Dudin, M. Hoesch, T. Kim, M. Radovic, M. Shi, Nicholas C. Plumb, C. Krellner, and Denis V. Vyalikh, Spin orientation of two-dimensional electrons driven by temperaturetunable competition of spin-orbit and exchange-magnetic interactions, Nano Lett. 17, 811 (2017).

[35] A. Chikina, A. Generalov, K. Kummer, M. Güttler, V. N. Antonov, Yu. Kucherenko, K. Kliemt, C. Krellner, S. Danzenbächer, T. Kim, P. Dudin, C. Geibel, C. Laubschat, and D. V. Vyalikh, Valence instability in the bulk and at the surface of the antiferromagnet $\mathrm{SmRh}_{2} \mathrm{Si}_{2}$, Phys. Rev. B 95, 155127 (2017).

[36] C. Klingner, C. Krellner, M. Brando, C. Geibel, F. Steglich, D. V. Vyalikh, K. Kummer, S. Danzenbächer, S. L. Molodtsov, C. Laubschat, T. Kinoshita, Y. Kato, and T. Muro, Evolution of magnetism in $\mathrm{Yb}\left(\mathrm{Rh}_{1-x} \mathrm{Co}_{x}\right)_{2} \mathrm{Si}_{2}$, Phys. Rev. B 83, 144405 (2011).

[37] M. Vijayakumar and M.S. Gopinathan, Spin-orbit coupling constants of transition metal atoms and ions in density functional theory, J. Mol. Struct.: THEOCHEM 361, 15 (1996).

[38] K. Kliemt and C. Krellner, Single crystal growth and characterization of $\mathrm{GdRh}_{2} \mathrm{Si}_{2}$, Journal of Crystal Growth 419, 37 (2015).

[39] D. V. Vyalikh, S. Danzenbächer, Yu. Kucherenko, K. Kummer, C. Krellner, C. Geibel, M. G. Holder, T. K. Kim, C. Laubschat, M. Shi, L. Patthey, R. Follath, and S. L. Molodtsov, $k$ Dependence of the Crystal-Field Splittings of $4 f$ States in Rare-Earth Systems, Phys. Rev. Lett. 105, 237601 (2010).

[40] S. Patil, K. Kummer, A. Hannaske, C. Krellner, M. Kuhnt, S. Danzenbächer, C. Laubschat, C. Geibel, and D. Vyalikh, Crystalline electric field splitting of $4 f$ states in $\mathrm{YbIr}_{2} \mathrm{Si}_{2}$ : An ARPES view, J. Phys. Soc. Jpn. 3, 011001 (2014).

[41] S. Patil, A. Generalov, M. Güttler, P. Kushwaha, A. Chikina, K. Kummer, T. C. Rödel, A. F. Santander-Syro, N. CarocaCanales, C. Geibel, S. Danzenbächer, Yu. Kucherenko, C. Laubschat, J. W. Allen, and D. V. Vyalikh, ARPES view on surface and bulk hybridization phenomena in the antiferromagnetic Kondo lattice $\mathrm{CeRh}_{2} \mathrm{Si}_{2}$, Nat. Commun. 7, 11029 (2016).

[42] K. Tomala, J. P. Sanchez, and R. Kmiec, Magnetic and crystal field properties of $\mathrm{DyRh}_{2} \mathrm{Si}_{2}$ and $\mathrm{ErRh}_{2} \mathrm{Si}_{2}$ from 161Dy and 166Er Mössbauer spectroscopy, J. Phys.: Condens. Matter 1, 9231 (1989).

[43] Z. Hossain, C. Geibel, F. Weickert, T. Radu, Y. Tokiwa, H. Jeevan, P. Gegenwart, and F. Steglich, Yb-based heavy-fermion metal situated close to a quantum critical point, Phys. Rev. B 72, 094411 (2005).

[44] H. Q. Yuan, M. Nicklas, Z. Hossain, C. Geibel, and F. Steglich, Quantum phase transition in the heavy-fermion compound $\mathrm{YbIr}_{2} \mathrm{Si}_{2}$, Phys. Rev. B 74, 212403 (2006).

[45] S. Friedemann, S. Wirth, N. Oeschler, C. Krellner, C. Geibel, F. Steglich, S. MaQuilon, Z. Fisk, S. Paschen, and G. Zwicknagl, Hall effect measurements and electronic structure calculations on $\mathrm{YbRh}_{2} \mathrm{Si}_{2}$ and its reference compounds $\mathrm{LuRh}_{2} \mathrm{Si}_{2}$ and $\mathrm{YbIr}_{2} \mathrm{Si}_{2}$, Phys. Rev. B 82, 035103 (2010).

[46] C. Krellner, S. Taube, T. Westerkamp, Z. Hossain, and C. Geibel, Single-crystal growth of $\mathrm{YbRh}_{2} \mathrm{Si}_{2}$ and $\mathrm{YbIr}_{2} \mathrm{Si}_{2}, \mathrm{Phil}$. Mag. 92, 2508 (2012).

[47] M. Hoesch, T. K. Kim, P. Dudin, H. Wang, S. Scott, P. Harris, S. Patel, M. Matthews, D. Hawkins, S. G. Alcock, T. Richter, J. J. Mudd, M. Basham, L. Pratt, P. Leicester, E. C. Longhi, A. Tamai, and F. Baumberger, A facility for the analysis of the electronic structures of solids and their surfaces by synchrotron radiation photoelectron spectroscopy, Rev. Sci. Instrum. 88, 013106 (2017).

[48] S. V. Borisenko, "One-cubed” ARPES User Facility at BESSY II, Synchrotron Radiation News 25, 6 (2012).

[49] D. V. Vyalikh, S. Danzenbächer, C. Krellner, K. Kummer, C. Geibel, Yu. Kucherenko, C. Laubschat, M. Shi, L. Patthey, R. Follath, and S. L. Molodtsov, Tuning the dispersion of $4 f$ bands in the heavy-fermion material $\mathrm{YbRh}_{2} \mathrm{Si}_{2}$, J. Electron Spectrosc. Relat. Phenom. 181, 70 (2010).

[50] P. E. Blöchl, Projector augmented-wave method, Phys. Rev. B 50, 17953 (1994).

[51] G. Kresse and J. Furthmüller, Efficient iterative schemes for ab initio total-energy calculations using a plane-wave basis set, Phys. Rev. B 54, 11169 (1996).

[52] G. Kresse and D. Joubert, From ultrasoft pseudopotentials to the projector augmented-wave method, Phys. Rev. B 59, 1758 (1999).

[53] John P. Perdew, K. Burke, and M. Ernzerhof, Generalized Gradient Approximation Made Simple, Phys. Rev. Lett. 77, 3865 (1996).

[54] D. D. Koelling and B. N. Harmon, A technique for relativistic spin-polarised calculations, J. Phys. C: Sol. St. Phys. 10, 3107 (1977).

[55] X. Zhang, Q. Liu, Jun-Wei Luo, Arthur J. Freeman, and A. Zunger, Hidden spin polarization in inversion-symmetric bulk crystals, Nat. Phys. 10, 387 (2014).

[56] A. S. Kutuzov, A. M. Skvortsova, S. I. Belov, J. Sichelschmidt, J. Wykhoff, I. Eremin, C. Krellner, C. Geibel, and B. I. Kochelaev, Magnetic susceptibility of $\mathrm{YbRh}_{2} \mathrm{Si}_{2}$ and $\mathrm{YbIr}_{2} \mathrm{Si}_{2}$ the basis of a localized $4 f$ electron approach, J. Phys.: Condens. Matter 20, 455208 (2008).

[57] K. Kummer, Yu. Kucherenko, S. Danzenbächer, C. Krellner, C. Geibel, M. G. Holder, L. V. Bekenov, T. Muro, Y. Kato, T. Kinoshita, S. Huotari, L. Simonelli, S. L. Molodtsov, C. Laubschat, and D. V. Vyalikh, Intermediate valence in $\mathrm{Yb}$ compounds probed by $4 f$ photoemission and resonant inelastic X-ray scattering, Phys. Rev. B 84, 245114 (2011).

[58] P. M. C. Rourke, A. McCollam, G. Lapertot, G. Knebel, J. Flouquet, and S. R. Julian, Magnetic-Field Dependence of the $\mathrm{YbRh}_{2} \mathrm{Si}_{2}$ Fermi Surface, Phys. Rev. Lett. 101, 237205 (2008).

[59] S. Danzenbächer, D. V. Vyalikh, K. Kummer, C. Krellner, M. Holder, M. Höppner, Yu. Kucherenko, C. Geibel, M. Shi, L. Patthey, S. L. Molodtsov, and C. Laubschat, Insight into the $f$-Derived Fermi Surface of the Heavy-Fermion Compound $\mathrm{YbRh}_{2} \mathrm{Si}_{2}$, Phys. Rev. Lett. 107, 267601 (2011).

[60] M. S. Dresselhaus, G. Dresselhaus, and A. Jorio, Group Theory. Application to the Physics of Condensed Matter (Springer Berlin Heidelberg, 2008).

[61] L. Elcoro, B. Bradlyn, Z. Wang, Maia G. Vergniory, J. Cano, C. Felser, B. Andrei Bernevig, D. Orobengoa, G. de la Flor, and Mois I. Aroyo, Double crystallographic groups and their representations on the Bilbao Crystallographic Server, J. Appl. Crystallogr. 50, 1457 (2017).

[62] P. Zhang, P. Richard, T. Qian, Y.-M. Xu, X. Dai, and H. Ding, A precise method for visualizing dispersive features in image plots, Rev. Sci. Instrum. 82, 043712 (2011).

[63] K. Kummer, S. Patil, A. Chikina, M. Güttler, M. Höppner, A. Generalov, S. Danzenbächer, S. Seiro, A. Hannaske, C. Krellner, Yu. Kucherenko, M. Shi, M. Radovic, E. Rienks, G. Zwicknagl, K. Matho, J. W. Allen, C. Laubschat, C. Geibel, and 
D. V. Vyalikh, Temperature-Independent Fermi Surface in the Kondo Lattice $\mathrm{YbRh}_{2} \mathrm{Si}_{2}$, Phys. Rev. X 5, 011028 (2015).

[64] Q. Y. Chen, D. F. Xu, X. H. Niu, J. Jiang, R. Peng, H. C. Xu, C. H. P. Wen, Z. F. Ding, K. Huang, L. Shu, Y. J. Zhang, H. Lee, V. N. Strocov, M. Shi, F. Bisti, T. Schmitt, Y. B. Huang, P. Dudin, X. C. Lai, S. Kirchner, H. Q. Yuan, and D. L. Feng, Direct observation of how the heavy-fermion state develops in CeCoIn ${ }_{5}$, Phys. Rev. B 96, 045107 (2017).

[65] D. Leuenberger, J. A. Sobota, S.-L. Yang, H. Pfau, D.-J. Kim, S.-K. Mo, Z. Fisk, P. S. Kirchmann, and Z.-X. Shen,
Dehybridization of $f$ and $d$ states in the heavy-fermion system $\mathrm{YbRh}_{2} \mathrm{Si}_{2}$, Phys. Rev. B 97, 165108 (2018).

[66] A. Yasui, Y. Saitoh, S.-I. Fujimori, I. Kawasaki, T. Okane, Y. Takeda, G. Lapertot, G. Knebel, T. D. Matsuda, Y. Haga, and H. Yamagami, Observation of bulk band dispersions of $\mathrm{YbRh}_{2} \mathrm{Si}_{2}$ using soft $\mathrm{x}$-ray angle-resolved photoemission spectroscopy, Phys. Rev. B 87, 075131 (2013).

[67] Shin-ichi Fujimori, Band structures of $4 \mathrm{f}$ and $5 \mathrm{f}$ materials studied by angle-resolved photoelectron spectroscopy, J. Phys.: Condens. Matter 28, 153002 (2016). 